\title{
Academic and Daily Life Satisfaction of Monbukagakusho Scholarship Students in Japan: The Case of Peruvian as International Students in Japan
}

\author{
Jakeline Lagones $^{1}$ \\ ${ }^{1}$ College of Foreign Studies, Kansai Gaidai University, Osaka, Japan \\ Correspondence: Jakeline Lagones, College of Foreign Studies, Kansai Gaidai University, 16-1 \\ Nakamiyahigashino-cho, Hirakata City, Osaka 573-1001, Japan. Tel: 81-72-805-2801. E-mail: \\ jlagones@kansaigaidai.ac.jp
}

Received: July 21, 2019

Accepted: August 12, 2019 Online Published: August 30, 2019

doi:10.5539/ass.v15n9p53

URL: https://doi.org/10.5539/ass.v15n9p53

\begin{abstract}
As the birth rate in Japan continues to decline, there is a growing need to ensure the availability of highly qualified foreign professionals. In order to attract more international students, it is necessary to understand the variables from the academic and daily life that international students face in Japan. This study shows the variables that influence academic satisfaction, daily life satisfaction and the relationship between these variables for Peruvian students serving as Monbukagakusho scholars in Japan. This study also describes the Monbukakusho scholars experience in their academic and daily life in Japan, which has a unique culture. Mix methodology was used in this study. The quantitative model based on individual data taken from a survey administered to Peruvian Monbukagakusho students, who were studying or are continuing their studies in Japan. The results show that the dependent variables and academic satisfaction are significantly related to language difficulties. Regarding the second dependent variable of, daily life satisfaction, we find a significant relationship between those who choose to study in Japan for cultural reasons and those, who study in shared laboratories and the type of scholarship received. Economic factors such as financial scholarship values are not related to dependent variables. A qualitative design of grounded theory was used as a framework to explore the experiences of Monbukagakusho students' definition of academic and daily life satisfaction in Japan. Five relevant Japanese codes stand out from the interviews; cohai-sempai, tatemae, honne, omoiyari and aisowarai.
\end{abstract}

Keywords: academic satisfaction, daily life satisfaction, international students, Monbukagakusho, scholarships, Peruvian students

\section{Introduction}

\subsection{Background}

According to a report presented by the MEXT (Ministry of Education, Culture, Sport, Science and Technology of Japan), the number of international students studying in Japan in the 2017 academic year amounted to approximately 276,000 with 9,166 sponsored by the Japanese government. Most foreign students awarded scholarships by the Japanese government originated from Asian countries such as China, accounting for students 1064 students in the 2017 academic year. The 2008 "Plan for 300,000 Foreign Students", created as a target for the year 2020 is sponsored by the Japanese government. The plan's objective is to render Japan more open to the rest of the world. As the birth rate in Japan continues to decline, there is a growing need to ensure the availability of highly qualified foreign professionals. The policy was also developed to promote the participation of young people and women in Japanese society and to sustain the country's future development. As such, based on the "Japan Recovery Strategy" and the "Second Plan for the Promotion of Basic Education" (Cabinet Decision on June 14th, 2013), it was decided that the number of international students should increase to 300,000 by 2020 . (Minister of Education Culture, Sports, Science and Technology-Japan [MEXT], 2018). Therefore, it is important to determine how international students overcome social, academic and cultural changes to their daily and academic lives as foreign students in Japan while pursuing their academic goals at Japanese universities. Monbukagakusho Scholarships are offered to applicants in more than 168 countries where application characteristics and programs developed are largely similar. Accordingly, this study analyzes the case of Peruvian 
Monbukagakusho scholars in Japan. International students in Japan needs to understand the adjustment problems faced by other international students experienced in Japan to give support. Based on this assumption, the objective of this study is to find the variables that influence Academic Satisfaction, Daily Life Satisfaction and the relationship between these variables for Peruvian students serving as Monbukagakusho scholars in Japan. In addition, how Monbukakusho scholars experience their academic and daily life in Japan which is ignored in existing research.

\subsection{Research Statement, Objective and Questions}

The 2008 "Plan for 300,000 Foreign Students", created as a target for the year 2020 is sponsored by the Japanese government. The plan's objective is to render Japan more open to the rest of the world. As the birth rate in Japan continues to decline, there is a growing need to ensure the availability of highly qualified foreign professionals. The policy was also developed to promote the participation of young people and women in Japanese society and to sustain the country's future development. As such, based on the "Japan Recovery Strategy" and the "Second Plan for the Promotion of Basic Education" (Cabinet Decision on June 14th, 2013), it was decided that the number of international students should increase to 300,000 by 2020. (Minister of Education Culture, Sports, Science and Technology-Japan [MEXT], 2018). Therefore, it is important to determine how international students overcome social, academic and cultural changes to their daily and academic lives as foreign students in Japan while pursuing their academic goals at Japanese universities. However, the literature does not present current data on international students studying in developed countries with special social codes such as Japan, which is socially, culturally and economically distinct from other developed countries. There has been limited research on the experiences of foreign students in Japan and especially regarding their levels of Academic Satisfaction and Daily Life Satisfaction. Accordingly, the study used a mixed methodology to answer the following questions; first; Which variables influence the daily lives and Academic Satisfaction of Peruvian Monbukagakusho scholars most in their pursuit of academic objectives in Japan?; second: What is the relationship between Daily Life Satisfaction and Academic Satisfaction for Peruvian Monbukagakusho scholars?; third; How Monbukakusho scholars experience their academic and daily life in Japan?. The hypothesis of this study is that statistically significant variables explain the daily life and academic satisfactions of Peruvian scholar and there is a relationship between "daily life satisfaction" and "academic satisfaction" for Peruvian Monbukagakusho scholars in their pursuit of their academic goals in Japan. The objective of my study is to analyze the variables that influence Monbukagakusho students' academic lives in Japan and levels of satisfaction generated by these variables throughout their stay in Japan as international students (referring to Peruvian Monbukagakusho scholars). The studied variables may improve the daily lives and academic experiences of future student scholars with Monbukagakusho Scholarships studying in Japan. This paper will be organized as follows: section two is dedicated to the literature review; the third section describes the research methodology; section four presents the quantitative analyzes and results; section fifth present the qualitative analyzes and results; and the last section presents the conclusion.

\section{Literature Review}

\subsection{Peruvians in Japan and the Mombukagakusho Scholarship}

Most Peruvians living in Japan can be divided into two groups. The first group includes Peruvian Nikkei who entered Japan with Japanese descendent visas. "Their purpose was to work, save money and return to Peru. However, after two decades, they continue working and residing in Japan with their families" (Lagones, 2016, p. 1). The other group composed of Peruvian international students, upon whom this article focuses. Although studying as a Peruvian Monbukagakusho fellow will be very different from studying as a Peruvian resident alien, those who do not have visa problems but are immersed in the Japanese educational system. However, Lagones (2015) noted that "Japan is not prepared at all for foreign residents. Japanese school law accepts any foreign children to enter the elementary and junior high school as Japanese children do, but all the lessons are taught only in Japanese" (p. 268). In the case of Peruvian as international students, lessons are taught in English, compare with the other ones who are residents in Japan. Therefore, the main difficulty that they encounter relates to financial problems and applications to student visas that allow them to continue with their academic objectives. Applicants for the Monbukagakusho Scholarship received support in these ones, but they are willing to face considerable challenges related to living in a country that is culturally, religiously and socially different.

First, there are a variety of programs for studying in Japan, that can be completed with grants from the Japanese government (e.g., the Monbukagakusho Scholarship) designed for foreign students with private funding or through short-term programs. Monbukagakusho Scholarships are offered to applicants in more than 168 countries where application characteristics and programs developed are largely similar. Therefore, the sample of 
recipients is homogenous, and small groups of students are small from each country enter the Monbukagakusho program each year.

The present work will focus on scholarships offered by the Japanese government to Peruvian students through its MEXT. The six scholarships offered include postgraduate scholarships, undergraduate scholarships, technical scholarships, occupational scholarships, teacher training scholarships and scholarships of the Japanese language and culture. These scholarships are unique in that, unlike others, are full scholarships, thus allowing students to focus entirely on their studies without financial concerns. Scholarships from the Japanese government are offered to countries with diplomatic relations with the Japanese government. The requirements for each scholarship can be found on the website of each cooperating embassy.

\subsection{International Students' Issues}

According to a report presented by the MEXT (Ministry of Education, Culture, Sport, Science and Technology of Japan), the number of international students studying in Japan in the 2017 academic year amounted to approximately 276,000 with 9,166 sponsored by the Japanese government. Most foreign students awarded scholarships by the Japanese government originated from Asian countries such as China, accounting for students 1064 students in the 2017 academic year.

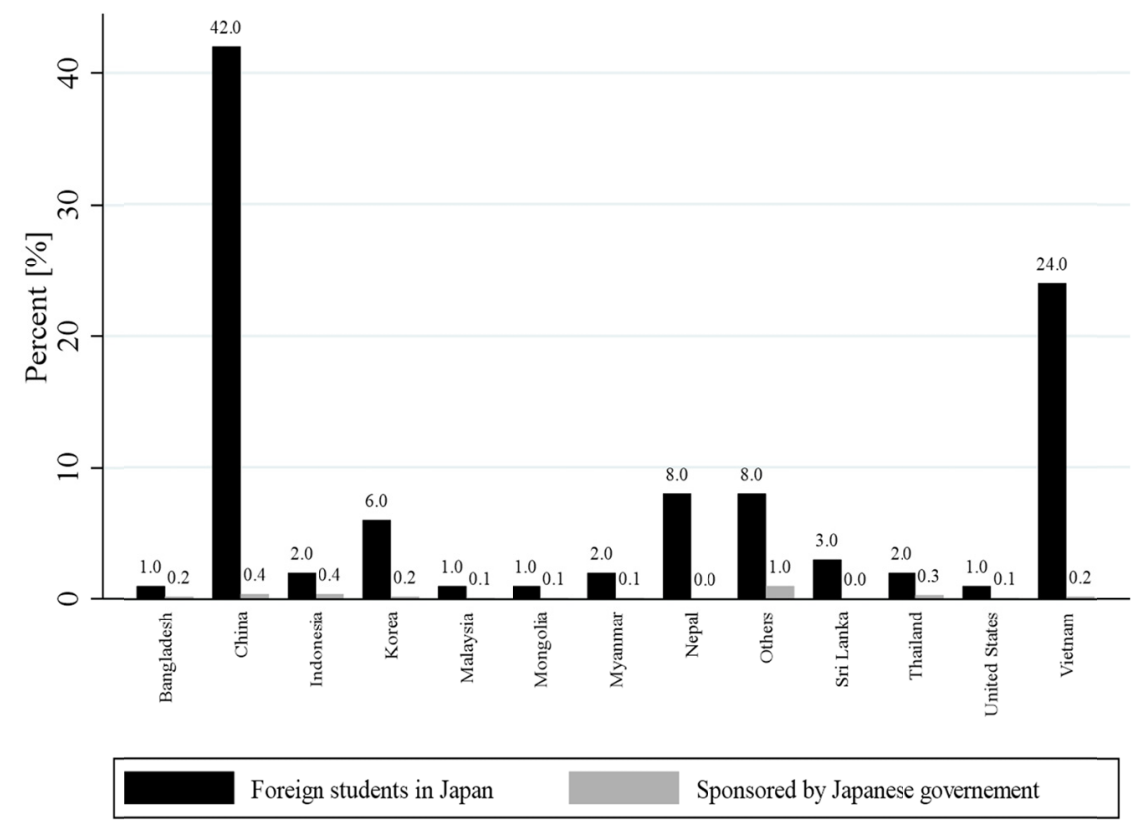

Figure 1. Foreign students in Japan in 2017

There has been limited research on the experiences of foreign students in Japan and especially regarding their levels of Academic Satisfaction and Daily Life Satisfaction. Nilsson (2015) confirmed that "Life satisfaction is an indicator of students' wellbeing. Life satisfaction has an indirect effect on student mobility and is often an invisible problem" (p. 90). "A Well-being international students life included two general categories, one of personal satisfaction and positive effect, the other related to pursuing a meaningful and successful study abroad" (Wen-Chic \& Newton, 2002). Therefore, "The necessity for social and cultural adaptation has a large impact on peoples daily live" (Hsu, 2011, p. 25).

On the other hand, another study examined the experiences of international students, scholars, and non-scholars in other countries and revealed problems related to such students' sense of belonging and to linguistic and cultural barriers among other issues throughout their adaptation to the host country. Yan and Pei (2018), analyzed the negative experiences of international students at American universities on and off-campus in their efforts to secure a sense of belonging. The results show that "students feel a sense of belonging with individuals from their home countries or similar ethnic groups... and in some cases, they accept the mistreatment of their teachers, not wanting to jeopardize their academic position, necessary to retain their visa" (p. 466). In a similar way, Horne et al. (2018) explain that "international students have much lower levels of belonging ... and this is felt among the highest performing students" (p. 365). Other results have found the following: "international students faced both linguistic and cultural barriers in their social interactions with American students" (Li \& Zizzi, 2018, p. 389). A 
clear example of this trend is observed in the United States, for which the findings reveal that international students face various challenges. Such students must deal with new people, societal customs, school systems and psychological and behavioural changes in adapting to their new environment. Such findings pertain to academic, social and cultural factors (Wu, Garza, \& Guzman, 2015).

Liu, in his research on international students' decisions to move to Japan, found that an interest in Japanese culture has a positive effect on international students' decisions to stay in Japan. On the other hand, Liu (2016) noted that language barriers serve as the most significant obstacle to this goal (p. 8). In 2003, Tamaoka, Ninomiya, and Nakaya concluded, "Japanese language ability is not necessarily required to communicate with supervisors and in conducting research" (p. 125). However, it should also be considered that the objective of studying abroad is to "gain experiences in the host country rather than abandoning its cultural heritage and adopting a cultural one where international students live for a relatively short period (Forbes-Mewett \& Nyland, 2008; Kingston \& Forland, 2008), where language constitutes an important part of this process" (cited in Newsome, 2016, p. 196).

In general, "Half of all international students go to five English-speaking countries: Australia, Canada, the United States of America, New Zealand and the United Kingdom" (UNESCO, 2018, p. 42). Additionally, "in several Asian countries with declining birth rates and ageing populations, such as Japan, higher education opens to foreign students" (UNESCO, 2018, p. 42). Therefore, it is important to consider that Japanese universities have a hierarchy- based system that plays an important role in academic relationships for foreign students in Japan. Researches have explained that the role of the academic advisor and the personal tutor as social support. (Yamamoto et al., 1986; Hicks, 1988).

These differences and the lofty academic goals that students pursue are limited when there are no academic resources available to inform them of the changes that come with becoming an international student in Japan. Therefore, it is vital to not only determine the benefits that this scholarship offers but to also identify the limitations and experiences of scholars and former grantees of the Monbukagakusho Scholarship in helping award recipients achieve a satisfactory academic experience and a satisfactory way of life while studying in Japan.

Specifically, four major categories emerged from the previous studies that will be analyzed to explain the levels of Academic Satisfaction and Daily Life Satisfaction of foreign students: (1) academic relationship; (2) daily life troubles;(3)motivation for the host country; (4) personal characteristics; and (5)economic.

\section{Research Methodology}

To test the hypothesis and answer the research questions; the study employs data from a mixed-method approach to research. For the quantitative analysis, I used the responses from the Dichotomous and rating scale questions (Closed-Ended Format) as an input to estimate an ordinal regression model. For the qualitative study, I used a deep interview with current Monbukagakusho students to explain their experiences of while they stay in Japan.

\subsection{Data Collection}

Data used for the present investigation were provided by student scholars and former grantees who have been recipients of the Monbukagakusho Japanese Scholarship (MOJAS). Surveys of Peruvian Monbukagakusho Scholarship students (SMSPS-Japan) were administered using Google online services, via email. According to Meho (2006), online communication can support some interviewers to express themselves (p. 1289). The SMSPS-Japan survey was designed by the researcher to identify and document experiences with the MOJAS before and during their studies in Japan to identify the variables that most heavily influence satisfaction with the MOJAS program in terms of academic and Daily Life Satisfaction and in terms of obtaining international student certification in Japan. The survey used semistructured open- and close-ended questions. The investigation also used qualitative data derived from interviews to clarify and support the quantitative results.

\subsection{Data Description}

Hill et al. (2005) recommend that sample from homogeneous composition could be selected randomly. A total of 20 scholars and ex-grantees of Monbukagakusho scholarships who had completed their studies in Japan were studied. The participants have the following characteristics: (a) Peruvian nationality and (b) past scholars and ex-grantees Monbukagakusho scholarships from the Japanese government. The 20 participants are Peruvians; $60 \%$ are men and $40 \%$ are women; $95 \%$ are single and 5\% are married without children; they are aged 19 to 33 years; $23.8 \%$ are Japanese descendants (Nikkei), and $76.2 \%$ are non-Nikkei Peruvians. Regarding academic focuses, $38.1 \%$ studied science, $28.6 \%$ studied the humanities, $28.6 \%$ studied engineering and $3.7 \%$ studied medicine. Regarding types of scholarships received, $71.4 \%$ of the students received postgraduate scholarships, $9.5 \%$ 
received undergraduate scholarships, 9.5\% received teacher training scholarships, $4.8 \%$ received technical scholarships and $4.8 \%$ received Japanese and culture scholarships. It is worth noting that regarding the students' activities in Peru before entering the scholarship program, $61.9 \%$ were working and studying, $19 \%$ were only studying and $19 \%$ were only working. In addition, $25 \%$ of the respondents had previously travelled to Japan for various reasons. Studies were carried out in, Tokyo, Yamagata, Tsukuba, Kyoto, Osaka, Chiba, Sapporo, Fukuoka, Nagasaki, Kashima, Hiroshima and Nagoya. Regarding the students' Japanese language abilities, 71.4\% had not completed the Japanese Language Proficiency Test (JLPT) and only $28.6 \%$ had taken it. Of the group of respondents who did complete the Japanese exam, 16.7\% had completed Level N5, 33.3\% had completed Level N4, 33.3\% had completed Level N3 and 16.7\% had completed Level N2. Levels N1 and N5 of the official Japanese Language Proficiency Test (JLPT) respectively test for the highest- and lowest levels of proficiency. Regarding English language proficiency, 76.2\% had taken an international exam in English, and 23.8\% had not.

\section{Quantitative Analysis}

The present study examines the most important variables that affect the Academic Satisfaction of Peruvian international students in pursuit of their academic objectives in Japan and their levels of Daily Life Satisfaction using a quantitative method. I consider funds allocated through each scholarship, types of scholarships received levels of satisfaction with such scholarships and part-time work performed by the students in Japan, as economic factors that can strengthen academic levels of satisfaction and Daily Life Satisfaction among students in Japan.

Other factors may also influence students' levels of academic and Daily Life Satisfaction while studying in Japan. The first relates to the academic environment involved, as this would affect students' satisfaction with social networks in their daily and academic lives. The second concerns social problems experienced in daily and academic life. The motivation to study in Japan could also influence levels of academic and Daily Life Satisfaction. For example, some students may decide to study in Japan due to their interest in manga culture. Finally, the personal characteristics of the students are evaluated

\subsection{Description of Independent Variables}

The independent variables were obtained through the SMSPS-Japan survey and were grouped into 5 categories: Personal characteristics, Motivation, Daily Life, Academic and Economic. In this study, 10 models were estimated, of which 5 pertain to the level of

Academic Satisfaction while the rest pertain to Daily Life Satisfaction. For each model, only independent variables $\left(\boldsymbol{X}_{\boldsymbol{n}}\right)$ belonging to a certain category were used.

a. The first category of Personal Characteristics, measures the following variables:

- Gender: Indicates the participants' gender $(1=$ Female, $0=$ Male $)$,

- Marital Status: Indicates the participants' marital status $(1=$ Married, $0=$ Single $)$,

- Nikkei: Indicates whether the respondent is a Japanese descendant $(1=$ yes, $0=$ no),

- Age: Indicates the participant's age at the time of receiving the scholarship $(3=34-30,2=25-30,1=21-25$, $0=18-25)$,

- Major: Indicates the participants' university major $(3$ =Engineering, $2=$ Medicine, $1=$ Science, $0=$ Humanities).

b. The second category, namely, Motivation measures the following:

- Culture: Indicates whether the respondent wants to study in Japan for cultural reasons. $(1=$ yes, $0=$ no),

- Education: Indicates if the respondent was motivated to study in Japan in search of quality education $(1=$ yes, $0=$ no),

- Scholarship: Indicates if the respondent was motivated to study in Japan due to benefits provided by the scholarship $(1=$ yes, $0=$ no $)$,

-Work: Indicates whether the respondent was motivated to study in Japan due to future career prospects $(1=$ yes, $0=$ no),

c. The third category, Daily Life, measures the following variables:

- Culture Shock: Indicates if the respondent experienced problems related to experiencing a culture shock during his or her stay in Japan $(1=$ yes, $0=$ no $)$,

- Language Barrier: Indicates if the respondent experienced problems related to language barriers during his or her stay in Japan $(1=$ yes, $0=$ no),

- Friendship: Indicates whether the respondent experienced problems related to friendship during his or her stay in Japan $(1=$ yes, $0=$ no $)$, 
- Professor: Indicates if the respondent experienced problems related to his or her professors during his stay in Japan $(1=$ yes, $0=$ no $)$,

- Weather: Indicates whether the respondent experienced problems related to climatic patterns during his or her stay in Japan $(1=$ yes, $0=$ no $)$,

- Travel Abroad: Indicates if the respondent had the opportunity to travel abroad during his or her their stay in Japan $(1=$ yes, $0=$ no $)$.

d. The fourth category, Academic, measures the following variables:

- Relationship with professor: Indicates if the respondent experienced positive relationships with his or her professors $(0=$ Not Good, 1 = Fair, 2 = Good $)$,

- Office relationships: Indicates whether the respondent experienced positive relationships with the university's international office $(0=$ No Good, 1 = Fair, $2=$ Good $)$,

- Research Laboratory: Indicates if the respondent completed his or her studies at a shared or private laboratory $(0=$ Shared, 1 = Private $)$.

e. The fifth category, Economy, measures the following variables:

- Type of Scholarship: Indicates the type of scholarship received by the respondent $(0=$ Undergraduate, $1=$ Postgraduate, 2 = Teacher Training, 3 = Technical, 4 = Japanese Training),

- Scholarship satisfaction: Indicates if the respondent is satisfied with the benefits that the scholarship offers $(1=$ yes, $0=$ no),

- Scholarship amount: Indicates the monetary value of the scholarship received $(0=$ Up to $100,000 ¥, 1=$ $100,000 ¥ \sim 150,000 ¥, 2=150,000 ¥ \sim 200,000 ¥, 3=$ More than $200,000 ¥)$,

- Part Time Job: Indicates whether the respondent engaged in part-time work during his or her stay in Japan $(1=$ yes, $0=$ no).

The below Table 1 shows the five categories and the descriptive statistics of the variables used in this research work:

Table 1. List of Data

\begin{tabular}{|c|c|c|c|c|c|c|c|}
\hline \multicolumn{2}{|r|}{ Categories } & Variables & Obs & Mean & Std. Dev. & Min & Max \\
\hline \multirow{5}{*}{\multicolumn{2}{|c|}{ Personal Characteristics }} & Gender & 20 & 0.4 & 0.50 & 0 & 1 \\
\hline & & Marital status & 20 & 0.1 & 0.30 & 0 & 1 \\
\hline & & Nikkei & 20 & 0.25 & 0.44 & 0 & 1 \\
\hline & & Age & 20 & 0.6 & 0.50 & 0 & 1 \\
\hline & & Major & 20 & 1.25 & 1.16 & 0 & 3 \\
\hline \multirow{4}{*}{\multicolumn{2}{|c|}{ b. Motivation }} & Select_culture & 20 & 0.55 & 0.51 & 0 & 1 \\
\hline & & Select_education & 20 & 0.7 & 0.47 & 0 & 1 \\
\hline & & Select_scholarship & 20 & 0.85 & 0.36 & 0 & 1 \\
\hline & & Select_work & 20 & 0.2 & 0.41 & 0 & 1 \\
\hline \multirow{7}{*}{\multicolumn{2}{|c|}{ c. Daily Life }} & Trouble_culture & 20 & 0.55 & 0.51 & 0 & 1 \\
\hline & & Trouble_language & 20 & 0.65 & 0.48 & 0 & 1 \\
\hline & & Trouble_friend & 20 & 0.3 & 0.47 & 0 & 1 \\
\hline & & Trouble_professor & 20 & 0.3 & 0.47 & 0 & 1 \\
\hline & & Trouble_weather & 20 & 0.3 & 0.47 & 0 & 1 \\
\hline & & Travel_abroad & 20 & 0.65 & 0.48 & 0 & 1 \\
\hline & & Professor_relationship & 20 & 0.4 & 0.50 & 0 & 1 \\
\hline \multirow{2}{*}{\multicolumn{2}{|c|}{ d. Academic }} & Office_relationship & 20 & 0.15 & 0.36 & 0 & 1 \\
\hline & & Research_lab & 20 & 0.35 & 0.48 & 0 & 1 \\
\hline \multirow{4}{*}{\multicolumn{2}{|c|}{ e. Economic }} & Type_of_sch & 20 & 1.25 & 0.91 & 0 & 4 \\
\hline & & Scholarship_satisfaction & 20 & 0.8 & 0.41 & 0 & 1 \\
\hline & & Scholarship_amount & 20 & 0.15 & 0.48 & 0 & 2 \\
\hline & & Part_time & 20 & 0.5 & 0.51 & 0 & 1 \\
\hline
\end{tabular}

Source: author

\subsection{Description of Dependent variables}

The regression model used is the ordinal probit using the following dependent variables: 
$S_{a}$ : Level of Academic Satisfaction on a scale of 1 (not satisfied) to 5 (very satisfied).

$\mathrm{S}_{\mathrm{d}}$ : Satisfaction with daily life, on a scale of 1 (not satisfied) to 5 (very satisfied).

\subsection{The Regression Model: Ordinal Probit}

In order to understand the relationship between the dependent and independent variables, I used a regression model approach. Therefore, I chose the ordinal probit model because our dependent variables are categorical ordered variables, and the probit model has been widely used in previous studies.

The estimation equation used is as follows:

$$
\mathrm{S}_{\mathrm{a}}=\sum_{n=1}^{N} \alpha_{n} X_{n}=\alpha_{1} X_{1}+\cdots+\alpha_{n} X_{n}
$$

$\mathrm{S}_{\mathrm{a}}$ : The level of Academic Satisfaction (dependent variable)

$\alpha_{n}$ : Coefficient of the independent variable (n)

$X_{n}$ : Independent variable (n)

$\mathrm{N}$ : Number of variables by category (Personal characteristics, Motivation, Daily Life, Academic and Economic)

$$
\mathrm{S}_{\mathrm{d}}=\sum_{n=1}^{N} \alpha_{n} X_{n}=\alpha_{0} X_{0}+\cdots+\alpha_{n} X_{n}
$$

$\mathrm{S}_{\mathrm{d}}$ : Daily Life Satisfaction (dependent variable)

$\alpha_{n}$ : Coefficient of the independent variable (n)

$X_{n}$ : Independent variable (n)

$\mathrm{N}$ : Number of variables by category (Personal characteristics, Motivation, Daily Life, Academic and Economic)

\subsection{Quantitative Results and Discussion}

According to the first hypothesis, there should be statistically significant variables that explain the Daily Life and Academic Satisfaction of Peruvian Monbukagakusho scholars who pursue their academic goals in Japan based on, variables of four categories: Motivation, Daily life, Academic and Economic.

For Motivation, we find no significant variable in terms of Academic Satisfaction. However, "Selection of culture" presents a positive estimated coefficient with respect to Daily Life Satisfaction, showing that those who choose to study in Japan due to its culture, exhibit a higher level of Daily Life Satisfaction. Students who are interested in Japanese culture are more motivated to understand Japanese social codes in their daily lives.

Next, for Daily Life, "Trouble with language" presents a negative estimated coefficient with respect to Academic Satisfaction. Thus, students who stated that they did not experience problems with the Japanese language exhibited a higher level of Academic Satisfaction. This is partly the case because English proficiency in Japan is not high, as only certain Japanese are required to address this gap in Japan. Second, those who understand the local language develop a stronger understanding of Japanese social codes. For Daily Life Satisfaction, no significant variables were found.

For the Academic category, with respect to Academic Satisfaction, no significant variables were found. However, for daily satisfaction, a significant relationship was found for the "Research lab" variable. Thus, students working in shared laboratories with other students enjoy more academic networking opportunities, rendering them more satisfied with their daily environment. Most students speak English at the universities where they study, allowing them to form social networks based on information that will prove useful outside of and on university campuses.

Further, as most laboratory students are foreign, students do not need to practice Japanese social codes with them.

Furthermore, for the Economic category, with respect to Academic Satisfaction, no significant variables were found. Regarding Daily Life Satisfaction, the "Type of scholarship" granted is significantly related to Daily Life Satisfaction. In this case, Japanese language scholarship expressed a higher level of Daily Life Satisfaction than recipients of the other scholarships. Thus, students more proficient in Japanese are more independent outside of and within university campuses due to their language ability even when they cannot engage in social networking. As they are not immigrants and live in Japan for a limited period of time, they do not need to engage in social 
networking, besides doing so in their academic and daily lives throughout their limited stay. Proficiency in the Japanese language helps the students better understand Japanese social codes compared to other students.

Finally, results observed regarding Personal Characteristics are not significant for either dependent variable (Academic Satisfaction and daily life).

Stronger coefficients were found for students citing culture as motivation to study in Japan (1.36) and working in a shared laboratory while studying in Japan (-1.35). These two variables have a stronger impact on levels of Daily Life Satisfaction. Regarding Academic Satisfaction, the most impactful coefficient corresponds to Language barriers" (-1.24), revealing a strong relationship between knowledge of the Japanese language and levels of Academic Satisfaction.

Regarding the second hypothesis, we find a relationship between "Daily Life Satisfaction" and "Academic Satisfaction" for Peruvian Monbukagakusho scholars in pursuit of their academic goals in Japan.

Results obtained from probit ordinal models of the quantitative analysis for the estimation of Academic Satisfaction are presented in the table below.

Table 2. Probit Model Regression: Academic Satisfaction

\begin{tabular}{|c|c|c|c|c|c|c|}
\hline Categories & Variables & Model 1 & Model 2 & Model 3 & Model 4 & Model 5 \\
\hline \multirow{10}{*}{ Personal Characteristics } & Gender & $\begin{array}{l}-0.22 \\
-077\end{array}$ & & & & \\
\hline & & {$[0.70]$} & & & & \\
\hline & Marital_status & 0.41 & & & & \\
\hline & & {$[0.64]$} & & & & \\
\hline & Nikkei & 0.16 & & & & \\
\hline & & {$[0.81]$} & & & & \\
\hline & Age & -0.45 & & & & \\
\hline & & {$[0.38]$} & & & & \\
\hline & Major & -0.12 & & & & \\
\hline & & {$[0.57]$} & & & & \\
\hline \multirow{8}{*}{ Motivation } & Culture & & 0.21 & & & \\
\hline & & & {$[0.70]$} & & & \\
\hline & Education & & -0.28 & & & \\
\hline & & & {$[0.66]$} & & & \\
\hline & Scholarship & & -7.09 & & & \\
\hline & & & [0.99] & & & \\
\hline & Work & & 0.93 & & & \\
\hline & & & {$[0.25]$} & & & \\
\hline \multirow{12}{*}{ Daily Life } & Culture Shock & & & -0.40 & & \\
\hline & & & & {$[0.40]$} & & \\
\hline & Language Barrier & & & -1.24 & & \\
\hline & & & & {$[0.03]^{* *}$} & & \\
\hline & Friendship & & & 0.00 & & \\
\hline & & & & {$[0.82]$} & & \\
\hline & Professor & & & -0.51 & & \\
\hline & & & & {$[0.77]$} & & \\
\hline & Weather & & & 0.22 & & \\
\hline & & & & {$[0.45]$} & & \\
\hline & Travel_abroad & & & -1.14 & & \\
\hline & & & & {$[0.07]^{*}$} & & \\
\hline \multirow{6}{*}{ Academic } & Professor relationship & & & & 0.63 & \\
\hline & & & & & {$[0.26]$} & \\
\hline & Office relationship & & & & -6.51 & \\
\hline & & & & & {$[0.99]$} & \\
\hline & Research lab & & & & 0.35 & \\
\hline & & & & & {$[0.53]$} & \\
\hline
\end{tabular}




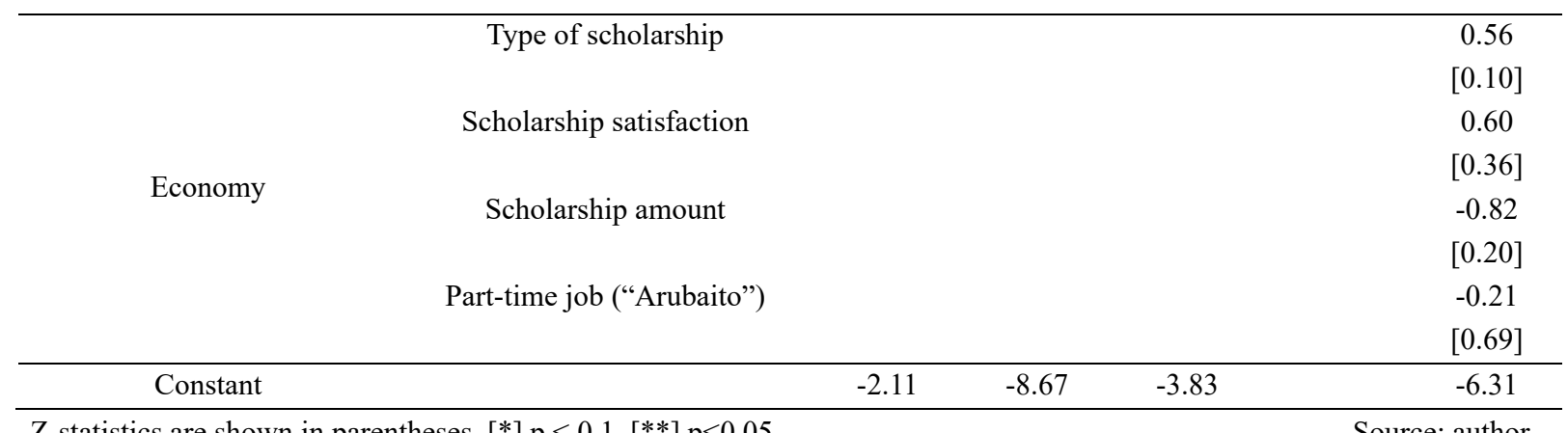

Z-statistics are shown in parentheses. [*] $\mathrm{p}<0.1,\left[{ }^{* *}\right] \mathrm{p}<0.05$.

Source: author.

Results obtained from the probit ordinal model of the quantitative analysis for the estimation of Daily Life Satisfaction are presented in the following table.

Table 3. Probit Model Regression: Daily Satisfaction

\begin{tabular}{|c|c|c|c|c|c|c|}
\hline Categories & Variables & Model 1 & Model 2 & Model 3 & Model 4 & Model 5 \\
\hline \multirow{10}{*}{ Personal Characteristics } & Gender & 0.77 & & & & \\
\hline & & {$[0.24]$} & & & & \\
\hline & Marital_status & 0.68 & & & & \\
\hline & & {$[0.46]$} & & & & \\
\hline & Nikkei & -0.22 & & & & \\
\hline & & {$[0.76]$} & & & & \\
\hline & Age & -0.50 & & & & \\
\hline & & {$[0.35]$} & & & & \\
\hline & Major & -0.46 & & & & \\
\hline & & {$[0.06]^{*}$} & & & & \\
\hline \multirow{8}{*}{ Motivation } & Culture & & 1.36 & & & \\
\hline & & & {$[0.03]^{* *}$} & & & \\
\hline & Education & & -0.73 & & & \\
\hline & & & {$[0.26]$} & & & \\
\hline & Scholarship & & -1.41 & & & \\
\hline & & & {$[0.17]$} & & & \\
\hline & Work & & 0.90 & & & \\
\hline & & & {$[0.27]$} & & & \\
\hline \multirow{12}{*}{ Daily Life } & Culture Shock & & & 0.26 & & \\
\hline & & & & {$[0.63]$} & & \\
\hline & Language Barrier & & & -0.05 & & \\
\hline & & & & {$[0.93]$} & & \\
\hline & Friendship & & & -0.24 & & \\
\hline & & & & {$[0.69]$} & & \\
\hline & Professor & & & -0.14 & & \\
\hline & & & & {$[0.83]$} & & \\
\hline & Weather & & & 0.42 & & \\
\hline & & & & {$[0.52]$} & & \\
\hline & Travel_abroad & & & -0.83 & & \\
\hline & & & & {$[0.15]$} & & \\
\hline \multirow{6}{*}{ Academic } & Professor relationship & & & & 0.13 & \\
\hline & & & & & {$[0.82]$} & \\
\hline & Office relationship & & & & -0.66 & \\
\hline & & & & & {$[0.40]$} & \\
\hline & Research lab & & & & -1.35 & \\
\hline & & & & & {$[0.03] * *$} & \\
\hline
\end{tabular}




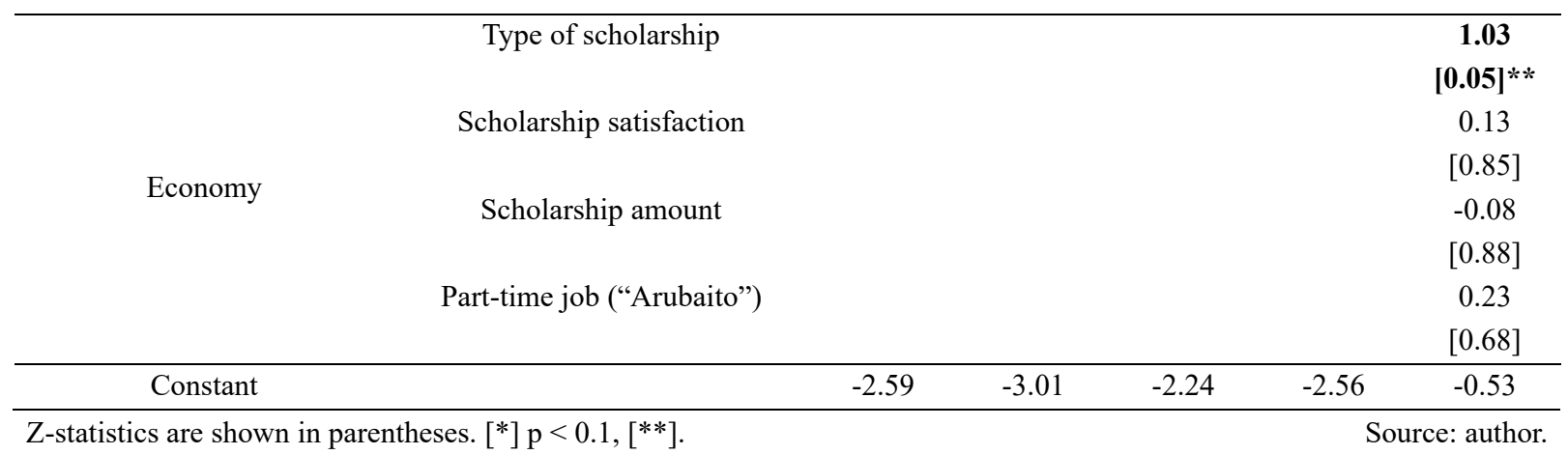

Results obtained from the probit ordinal model of the quantitative analysis for the estimation of Daily Life Satisfaction considering Academic Satisfaction as an independent variable and vice versa are presented in the following table.

Table 4 shows a strong correlation between "Daily Life Satisfaction" and "Academic Satisfaction." Therefore, if the independent variables enhance Academic Satisfaction, levels of Daily Life Satisfaction should also increase due to this correlation. In the same way, if the independent variables enhance Daily Life Satisfaction, Academic Satisfaction should increase as well.

Table 4. Probit Regression Model: Academic Satisfaction vs. Daily Life Satisfaction

\begin{tabular}{ccc}
\hline Variable & Daily Satisfaction & Academic Satisfaction \\
\hline Academic Satisfaction & 0.8468124 & \\
& {$[0.017]^{* *}$} & 0.9482428 \\
Daily Satisfaction & & {$[0.011]^{* *}$} \\
\hline Z-statistics are in shown parentheses. [*] $\mathrm{p}<0.1,[* *] \mathrm{p}<0.05$ & Source the author
\end{tabular}

\section{Qualitative Analyses}

The research, previously described, variables that explain the Daily Life and Academic Satisfaction of Peruvian Monbukagakusho scholars. Qualitative analysis is necessary to explain how Monbukakusho scholars experience their Academic and Daily Life in Japan. A qualitative design of grounded theory was used to analyze Monbukagakusho scholars' definition of Daily Life and Academic Satisfaction to figure out categories and variables that scholars as international students face in Japan. I will focus on finding academic and social codes that scholars face in Japan by asking the question: How do Monbukagakusho scholars define their Daily Life and Academic Satisfaction as a foreign student in Japan?

Five Monbukagakusho scholars who are studying at a Japanese public university participate in this research. The aim was to add a new approach from the Daily Life and Academic Satisfaction that Peruvian Monbukagakusho scholars experienced in Japan. The five interviewed are Peruvians, between twenty-five and thirty-four years old, one is married and four singles. The researcher obtains consent from each interviewer before the interview started. The interview took place in a public place in Aichi Prefecture Japan. The time expended in the interview differs one by one between one and two hours and it was tape-recorded.

\subsection{Data Analysis}

The analysis of the data used the grounded theory; (1) open coding (2) selective coding and (3) Axial coding. After the interviewee, the research uses the open coding, to transcript the tape-recorded by sentence analysis, to figure out the relevant categories. Then, during the axial coding process, the research figures out the subcategories to compare, expand and explore the variation in the phenomena. Finally, the selective coding process is used to select the central category.

\subsection{Qualitative Results and Discussion}

According to the quantitative results, there is a strong correlation between Academic and Daily Life Satisfaction. Therefore, a qualitative study from the interviewees described Monbukagakusho scholar experiences at Japanese universities regarding their Academic and Daily Life Satisfaction throughout their time in Japan. From the interviewees, two important issues were analyzed to examine how Peruvian Monbukagakusho students challenged (1) Academic Satisfaction and (2) Daily Life Satisfaction in Japan. First, interviewees define the 
concept of Daily and Academic Satisfaction, to detect their satisfaction in their academic and daily life while studying in Japan.

The interviewers reported many experiences regarding satisfaction in their academic and daily life. Then, open coding, axial coding and selecting coding of data analysis figure out two main categories: (1) Pursue academic goals for satisfaction and (2) Self-taught and independence for a satisfactory life as a foreign student.

\subsubsection{Pursue Academic Goals for Satisfaction}

According to the interviewees, their concepts of Academic Satisfaction defined in terms of its progression: The interviewees reported, "Academic Satisfaction is how you progress. I feel good when I continue with an academic speciality that I like and when I can contribute with patience. Satisfaction comes from doing something better." "Academic Satisfaction is achieved when I can meet my learning and academic development goals." "For example, Japan offers resources, equipment, etc., because everything related to engineering requires access to resources." "I try to understand and use Japanese academic rules and skills in order to obtain my academic goals."

From the previous description of academic satisfaction, the interviewee's outcome developed two important categories for obtaining academic satisfaction in Japan; (1) motivation for academic goals and (2) understanding new academic rules and skills from the host country (Japanese codes).

a. Academic motivation

If the academic motivation of students is low, it will be difficult for them to take advantages of the benefits of studying in a country developed as Japan.

One respondent, a student who studies engineering, reported, "The impact of science and engineering is global, and some Japanese universities focus on supporting projects to be applicated in developed countries." This characteristic link with the academic goals of some post-graduate international students too, "I want to manage a research laboratory and obtain funding for a project to help my home country." Other respondent reported, "Some Peruvian foreign students, who successfully finished their studies in Japan, found an academic position in Japan." The academic motivation will help Peruvian students to expand their knowledge through conferences in other countries while they are in Japan. "The capacity to travel to other countries, and advantages in terms of obtaining a visa from other countries."

\section{b. Academic rules: Cohai - Sempai relationships}

In Japan, unlike other countries, academic rules are singular. The terms sempai(first) and cohai (second) mean the hierarchy of respect that exists in Japan between the individual who has more experience and the one who has less experience without considering age. Therefore, considering the academic experience is relevant, the sempai is greater than cohai. The interviewees reported, "Learning is very hierarchical. A Master's student cannot say anything to a PhD student. First, I thought they did not want to say anything. When a PhD student is talking, the Cohai is silent. For a higher cycle, sometimes the teacher asks the student before entering a Cohai job." "In my case, I dedicate myself to mechanical and control system design. Sometimes there are several options, but what the teacher or sempai says must be applied. Even when there is a better approach, it is not used." "Here you adapt to the style of work because there is a friendly environment for successful work and cooperation in the laboratory." "When you attend classes, you must follow academic rules to pass academic courses in Japan." "Studying here to learn, Japan is better than studying in another developed country where I was before." "In the other developed country, they hope that you already know how to complete the academic work in advance." "In the other developed country, they give me the recipe, but they do not teach me as they do in Japan. Here in Japan, there is a lot of camaraderies, and everything is worked on as a team. In the other developed country, individualism is prioritized." "Positions in Peru segregate. This does not exist in Japan." "The sempai stands behind the cohai to determine what you are doing and teach you. They do this to guide you. At first, I did not understand it, but then I realized that the objective was to do this because how you are to work is pre-determined." I realized this later as I learned more about Japanese culture."

\subsubsection{Self-taught and Independence for a Satisfactory Life as a Foreign Student}

Regarding Daily Life Satisfaction the respondent reported:

"Daily Life Satisfaction can be defined as satisfaction with living fully in the social environment in general, family life, in academia, etc." other respondent reported, "Become independent before travelling to the host country helped me to assimilate local rules more easily for my Daily Life Satisfaction." In Japan it is not common to have a housekeeper, almost all Japanese even they have a job, they must do household by themselves. 
And the most difficult to learn for foreign people is the recycling garbage system. As students reported "I do not usually cook or clean in Peru. In Japan, I have to cook, clean, recycle and shop by myself." "In Aichi prefecture, I have to separate the trash in five types." "The public transportation system here and the security are convenient to compare to my country".

From the previous description of Daily Life Satisfaction, the interviewee's outcome developed four important categories for obtaining satisfaction in their daily life as international scholar; (1) motivation to understand the host culture, (2) developing a multicultural network, (3) basic Japanese language and (4) understanding social codes of the host country.

\section{a. Culture motivation}

Before foreign students came to Japan, they realized that Japan has an original culture that would have motivated them also. The interviewees reported, "I was highly motivated to come to Japan." "I came to study the academic subject more than to understand the culture, but on the way, I feel more motivated due to Japanese particular behaviour (always smiling, extremely kind, just never angry...)." These are because of Japanese codes called Aisowarai, Honne and Tatemae.

b. Multicultural networks

The interviewees reported, "Learning culture from different countries was one of my goals to become a global citizen, I could do it in Japan." "My multicultural network helped me to understand some social rules from different countries, and we could talk in English which is not the native language for any of my multicultural friends, this reduces my pressure in the language, and we felt comfortable." "I experienced initial difficulty because I did not know must about the country. I had read about Japan in books, but I had not lived in Japan, later I could understand that Japan has special cultural, academic and social codes that would be useful in my whole life." Regarding previous descriptions of multicultural networks, the respondents show a positive point for their daily life satisfaction as a foreign student in Japan.

c. Japanese language

Studies were completed in English, which is not their native language nor is it the native language for Japanese people. The interviewees reported, "Understanding the language is key to being successful in any countries, but I needed the Japanese language only for specific things as shopping." "The language was difficult to learn. I only knew the basics of hiragana and Katakana (Japanese alphabet), and the procedures were difficult, but my university tutors in charge of me and professors helped me." "At the hospital there were people who helped me, find an apartment, buy a cell phone and so on. There was camaraderie and they invited me to their parties as well. You do not feel culture shock because they help you, and they try to make your transition less traumatic." The Monbukagakusho grantees receive the lessons in English, which is not the native language of their Japanese teachers either. Therefore, the pressure of the language is not so complicated, but they need to use the basic Japanese language for daily life communication.

\section{d. Japanese Social codes}

In Japan, unlike other countries, the social and academic rules are unique, there are so-called Japanese social codes that, in order to be understood, must be lived in Japan, so I will describe the experiences of Peruvian students in Japan. The interviewees reported, "I listened that respect, discipline, responsibility and humility are more important than intelligence. So, I wanted to know how it is." "My personal life has benefitted in Japan from my Japanese colleagues' courtesy and collective mentality, which I did not understand at first." "I am currently comfortable at the laboratory; I am doing what I expected to do." "Social changes were experienced after my arrival." "If you do not know anything about Japanese culture, first, you isolate yourself. But then you understand that it would be important to learn about your life." "Work in collaboration is one Japanese characteristic that I learned" "Work for the benefit of the group more than individual success is the key to improve your nation." "I did not like the concepts of Tatemae and Honne very much, as it can be very difficult to understand Japanese people because they do not show what they think. Sometimes I thought that they wanted to say no but did not. I later processed this and can now understand my colleagues better." "When I was a research student, I liked to ask questions, and at first, they did not tell me the answer until the professor told me. In Perú, medicine is based on evidence and not on hierarchies or experience. Here, the emphasis on the hierarchy is sometimes correct, but not always." Another code is called Omoiyari which means: Not engaging in inappropriate actions

"For example, even if you speak only a little Japanese, they will say "You speak Japanese very well." "Sometimes their compliments sound somewhat sarcastic, and they do not sound sincere to us Latinos. They tell 
you at every moment, it's really about Omoiyari."

Honne and Tatemae which means inside and outside of your thinking, do not show outside your negative points. "They do not speak directly, and they do not tell you that something can be improved. They always tell you that it's okay." Another Japanese code is called Aisowarai. Smiling is very important to demonstrate kindness to others. One student reported "Sometimes, when I say something serious or mention a mistake, Japanese people smile. At first, I thought they were not listening to me, but they actually do this because they do not want to show that they are worried and create a negative environment even when there is a problem."

\section{Conclusions}

Regarding the first research question, the quantitative results of this study do not reveal significant effects of economic factors (a form of scholarship, scholarship satisfaction, scholarship amount, and part-time job) on Academic Satisfaction for Peruvian Monbukagakusho students. However, the type of scholarship received shapes student's Daily Satisfaction in Japan even when the scholarship earned provides less funding than other scholarships. Students working in a shared international research laboratory noted a positive effect on their levels of Daily Life Satisfaction because such involvement rendered them independent in their academic and social lives in Japan because outside of the international lab, students need to understand Japanese social and academic rules, as the interviewees explained. Having an interest in Japanese culture can have a positive effect on Daily Satisfaction with life in Japan. As some students corroborated it in the qualitative results (interviewees). On the other hand, difficulty with language barriers constituted a limitation to Academic Satisfaction for Peruvian Monbukagakusho scholars. But learning basic Japanese for communication would be enough, according to the qualitative results.

For the second research question, a strong correlation was found between "Daily Life Satisfaction" and "Academic Satisfaction." To enhance levels of satisfaction among Peruvian Monbukagakusho scholars, one of these two variables could be initially addressed.

According to the third research question, the interviewers reported many experiences regarding satisfaction in their academic and daily life. Then, open coding, axial coding and selecting coding of data analysis figure out two main categories: (1) Pursue academic goals for satisfaction: motivation for academic goals and understanding new academic rules and skills from the host country as Japanese codes. It is important for foreign scholars to understand Japan's uniqueness social and academic codes as the qualitative result described. (a) Cohai - Sempai relationships. The terms sempai(first) and cohai (second) mean the hierarchy of respect that exists in Japan between the individual who has more experience and the one who has less experience without considering age. Therefore, considering the academic experience is relevant, the sempai is greater than cohai. (b) Omoiyari refers to not engaging in inappropriate actions. (c) Honne and Tatemae which means inside and outside of your thinking, do not show outside your negative points. Another Japanese code is called (d) Aisowarai. Smiling is very important to demonstrate kindness to others. The second main category; (2) Self-taught and independence for a satisfactory life as a foreign student. The interviewee's outcome developed four important categories for obtaining satisfaction in their daily life as an international scholar; (a) motivation to understand the host culture, (b) developing a multicultural network, (c) basic Japanese language and (d) understanding social codes of the host country.

Based on this study's results, I recommend that more research is required in assessing other Japanese social codes experienced by international students in Japan.

\section{References}

Chirkov, V., Vansteenkiste, M., Tao, R., \& Lynch, M. (2007). The role of self-determined motivation and goals for study abroad in the adaptation of international students. International Journal of Intercultural Relations, 31(2), 199-222. https://doi.org/10.1016/j.ijintrel.2006.03.002

Hicks, J. (1988). Studies on the Adjustment of Foreign Students in Japan: With focus on Interpersonal Relations (Doctoral Dissertation). Hiroshima University, Hiroshima.

Hill, C. E., Knox, S., Thompson, B. J., Nutt W. E., Hess, S. A., \& Ladany, N. (2005). Consensual Qualitative Research: An update. Journal of Counseling Psychology, 52(2), 196-205. https://dx.doi.org/10.1037/0022-0167.52.2.196

Horne, V., Lin, S., Anson, M., \& Jacobson, W. (2018). Students Engagement, Satisfaction, and Belonging of International Undergraduates at U.S. Research Universities. Journal of International Students Engagement, 8, 351-374. https://doi.org/10.5281/zenodo.1134313 
Hsu, C. (2011). Factors Influencing International Students' Academic and Sociocultural Transition in an Increasingly Globalized Society (Doctoral Dissertation). 1-154. Available from ProQuest LLC. (UMI No. 3455443)

Lagones, J. (2015). Challenges of Nikkei Peruvian Second Generation in Japan: An Overview of Their Employment Status after the Lehman Shock. Asian Social Science, 11(26), 267-280. https://dx.doi.org/10.5539/ass.v11n26p267

Lagones, J. (2016). Socioeconomic Characteristics of First-Generation Nikkei Peruvians Who Remained in Japan During and After Lehman Shock (2009-2014). Forum of International Development Studies, 47(1), $1-19$.

Li, S., \& Zizzi, S. (2018). A Case Study of International Students Social Adjustment, Friendship Development, and Physical Activity. Journal of International Student, 8, 389-408. https://doi.org/10.5281/zenodo.1134317

Meho, L. (2006). E-Mail Interviewing in Qualitative Research: A Methodological Discussion. Journal of the American Society for Information Science and Technology, 57(10). https://doi.org/10.1002/asi.20416

Minister of Education Culture, Sports, Science and Technology Japan (MEXT). (2018). About the number of scholarship program. Retrieved from http:/www.mext.go.jp/a_menu/koutou/ryugaku/1338568.htm

Ministry of Education, Culture, Sports, Science and Technology (MEXT). (2018). International students. Retrieved from http://www.MEXT.go.jp/en/News/topics/detail/sdetail/1372647.htm

Newsome, 1. (2016). International Students Cultural and Social Experiences in a British University: "Such a hard life is here". Journal of International Students, 6(1), 195-215.

Nilsson, P. (2015). Life Satisfaction among Outbound Students in Northern Sweden. World Journal of Education, 5(4), 87-92. https://dx.doi.org/10.5430/wje.v5n4p87

Tamaoka, K., Ninomiya, A., \& Nakaya, A. (2003). What Makes International Students Satisfied with a Japanese University. Asia Pacific Education Review, 4(2), 119-128.

Tseng, W.-C., \& Newton, F. (2002). International students' strategies for well-being. College Student Journal, $36(4), 591$.

UNESCO, (2018). Global Education Monitoring Report Summary 2019: Migration, displacement and education: Building bridges, not walls. Paris, UNESCO.

Vasilopoulos, G. (2016). A Critical Review of International Students' Adjustment Research from a Deleuzian Perspective. Journal of International Students, 6(1), 283-307.

Wu, H., Garza, E., \& Guzman, N. (2015). International Students Challenge and Adjustment College. Education Research International, 1-9. https://dx.doi.org/10.1155/2015/202753

Yamamoto, T. (1986). An environmental psychological study of adjustment in a cross-cultural environment transition. Report of a Ministry of Education Grant, General Research Class B.

Yan, L., \& Pei, S. (2018). "Home Away from Home?" How International Students Handle Difficult and Negative Experiences in American Higher Education. Journal of International Students, 8(1), 453-472. https://dx.doi.org/10.5281/zenodo. 1134338

\section{Copyrights}

Copyright for this article is retained by the author(s), with first publication rights granted to the journal.

This is an open-access article distributed under the terms and conditions of the Creative Commons Attribution license (http://creativecommons.org/licenses/by/4.0/). 